\title{
Cementos de escorias activadas alcalinamente: Situación actual $y$ perspectivas de futuro
}

\author{
F. PUERTAS \\ Dra. en Ciencias Químicas IETCC/CSIC \\ ESPAÑA
}

Fecha de recepción: 27-V-95

\section{RESUMEN}

Se presenta el esiado del conocimiento actual de los cementos y hormigones de escorias activadas alcalinamente, detallándose las caracteristicas y propiedades de los citados materiales.

Se incide especialmente en aquellos aspectos de carácter científico y técnico que permanecen sin resolver y que, por tanto, precisan nuevas investigaciones.

\section{SUMMARY}

The paper presents the state of the art in cements and concretes with alkaline-activated slag. Characteristics and properties of these materials are given.

Special emphasis is made on those scientific and technical aspects which remain unsolved and which, therefore, call for further research.

\section{INTRODUCCIÓN}

El principio en el que se basa la activación alcalina de escorias fue propuesto en 1940 por Purdon(1). Pero fue en los años 60 cuando Glukhovsky et al $(2,3)$ desarrollaron los primeros cementos de escorias activadas alcalinamente. La primera planta piloto de producción de este tipo de cementos y hormigones se implantó en Ucrania en 1960, iniciándose la producción industrial de los citados materiales en 1964. A partir de los años 70 el interés por los cementos activados alcalinamente fue creciendo en otros países distintos de la antigua Unión Soviética, como en China (4,5), Europa occidental $(6,7,8)$, India (9), Japón (10), USA (11).

Pese aese interés continuado, en la actualidad, los cementos de escorias activadas alcalinamente, siguen siendo objeto de estudio por parte de la comunidad científica, ya que aún permanecen muchas incógnitas por resolver. No hay un conocimiento suficiente en torno a los mecanismos que controlan los procesos de activación de laescoria; asimismo, es necesario profundizar en el conocimiento de la naturaleza de los productos de hidratación formados y en la relación de estos con la sustancia activadora y con el posterior comportamiento mecánico-resistente de los cementos y hormigones formados (desarrollo de resistencias, retracción al secado, formación de microfisuras, durabilidad, etc.).

A continuación se va a presentar el estado del conocimiento en torno a los procesos de formación y propiedades de los cementos y hormigones de escorias activadas alcalinamente.

\section{ESCORIAS Y SUSTANCIAS ACTIVADORAS}

Los estudios realizados han demostrado que escorias de diferente origen y naturaleza pueden ser activadas alcalinamente (12) para formar productos de hidratación de naturaleza adhesiva y cementante. Son las escorias molidas granuladas de alto horno, aquellas que preferentemente suelen ser utilizadas para tal fin.

Sin embargo, también pueden ser empleadas escorias de electrotermo-fosforosas y otras escorias metalúrgicas.

La actividad hidráulica de las escorias depende fundamentalmente de su estructura, la cual, a su vez, está íntimamente relacionada con la composición química $\mathrm{e}$ historia térmica de dichas escorias. 
Las escorias de naturaleza básica, es decir, aquellas con relación $\mathrm{CaO}+\mathrm{MgO} / \mathrm{SiO}_{2}>1$, tienen mayor potencial hidráulico. Narangy Chopra(12) encontraron que escorias sintéticas con relaciones $\mathrm{CaO} / \mathrm{SiO}_{2} \simeq 1,38$ desarrollan, en su activación con $\mathrm{NaOH}$, resistencias mecánicas tres veces superiores a las desarrolladas por las escorias con relación $\mathrm{CaO} / \mathrm{SiO}_{2} \simeq 1,0$. El contenido de cal en la escoria controla la activación de la misma.

En las escorias fosfáticas, el $\mathrm{P}_{2} \mathrm{O}_{5}$ parece jugar un papel positivo en la activación de la escoria; por el contrario, el $\mathrm{Cr}_{2} \mathrm{O}_{3}$, en las escorias de aleación, muestra un efecto negativo en el desarrollo de resistencias, aunque el contenido de cal en la citada escoria sea relativamente elevado (12).

En las escorias molidas granuladas de alto horno se ha comprobado que la relación gelenita/akermanita en el vidrio de melilita tiene influencia sobre el proceso de activación (13). Las escorias ricas en gelenita $\left(C_{2} A S\right)$ desarrollan mayores resistencias en su activación. De cualquier modo, lo que estos autores si han encontrado es una relación directa entre la composición química de la escoria y la naturaleza del activador. Así, las escorias ricas en akermanita $\left(\mathrm{C}_{2} \mathrm{MS}_{2}\right)$ desarrollan mayores resistencias cuando son activadas con $\mathrm{Na}_{2} \mathrm{CO}_{3}$, mientras que las de gelenita lo hacen cuando reaccionan con el $\mathrm{NaOH}$.

Sin embargo, escorias de igual composición química pueden presentar diferente actividad hidráulica, ya que pueden poseer distintos contenidos en fase vítrea (14). La cantidad de fase vítrea en la escoria depende de la historia térmica experimentada por la misma.

Al enfriar bruscamente la escoria líquida o fundida se obtiene un vidrio de forma irregular y elevada energía interna (aproximadamente el calor de cristalización es de $200 \mathrm{~J} / \mathrm{g}$ ), lo que hace que sea un material altamente reactivo. Como es sabido en la fase vitrea de la escoria reside su componente hidráulicamente activo, pudiendo ser considerada la fase cristalina prácticamente como un inerte.

Según Zhang et al (15) la solubilidad de la escoria, y por lo tanto su reactividad frente al agua u otras sustancias de activación, depende del grado de polimerización de los tetraedros $\left(\mathrm{SiO}_{4}\right)$ y de la coordinación del $\mathrm{Al}$ y del $\mathrm{Mg}$ en la fase vítrea de la escoria. Cuanto mayor es el grado de polimerización en el vidrio menores suactividad hidráulica. Estos autores proponen la relación $\mathrm{MeO}_{6} / \mathrm{MeO}_{4}=0,5$ (donde $\mathrm{Me}=\mathrm{Al} \circ \mathrm{Mg}$ ) como la óptima para conseguir la máxima hidraulicidad.

Diferentes autores $(15,16,17,18)$ consideran que la presencia de una pequeña proporción de fase cristalina puede aumentar la hidraulicidad de la escoria, ya que esos núcleos cristalinos pueden incrementar el desorden estructural e impedir la polimerización de los tetraedros de silicio ó aluminio.

La superficie específica de la escoria tiene también una gran influencia sobre la velocidad e intensidad de sus reacciones de hidratación. Diferentes autores $(4,6,19,20$, 21) han indicado las superficies específicas óptimas de la escoria para alcanzar las máximas resistencias. Shi y Li (19) demuestran que superficies específicas por encima de $4.000 \mathrm{~cm}^{2} / \mathrm{g}$ no incrementan las resistencias a compresión a edades superiores a los 28 días. Para Wang (4) el rango óptimo de finura de la escoria se encuentra entre 4.000-5.500 $\mathrm{cm}^{2} / \mathrm{g}$; para Gjorv (6) ese rango se sitúa entre $4.200-6.000 \mathrm{~cm}^{2} / \mathrm{g}$. Otros autores (20) indican que ese rango óptimo de superficie específica debe de ser determinado atendiendo a factores no únicamente resistentes, sino también tecnológicos y económicos. A partir de sus resultados comprueban que la superficie específica óptima de la escoria depende de la naturaleza de la misma, de los activadores y de las condiciones de curado. Así, indican que la superficie específica óptima para las escorias ácidas ó neutras es de $4.500-6.500$ $\mathrm{cm}^{2} / \mathrm{g}$, mientras que en las escorias básicas esa superficie debería de estar entre $4.000-5.500 \mathrm{~cm}^{2} / \mathrm{g}$. Si las condiciones de curado son aceleradas, la efectividad del incremento de la finura de la escoria puede ser reducida.

En USA se han desarrollado unos cementos denominados "Slag ultrafine Cements" basados en mezclas de escorias de alto horno (75\%) y cemento portland (25\%) (21). En estos cementos, el tamaño máximo de las partículas es inferior a $10 \mu \mathrm{m}$.

Las sustancias activadoras son: compuestos alcalinos ó alcalinotérreos como hidróxidos ( $\mathrm{ROH}, \mathrm{R}(\mathrm{OH})_{2}$ ), sales de ácido débil $\left(\mathrm{R}_{2} \mathrm{CO}_{3}, \mathrm{R}_{2} \mathrm{~S}, \mathrm{RF}\right)$, sales de ácido fuerte $\left(\mathrm{Na}_{2} \mathrm{SO}_{4}, \mathrm{CaSO}_{4} \cdot 2 \mathrm{H}_{2} \mathrm{O}\right)$ y sales silícicas del tipo $\mathrm{R}_{2} \mathrm{O}$.(n) $\mathrm{SiO}_{2}$, donde $\mathrm{R}$ es un ión alcalino del tipo $\mathrm{Na}, \mathrm{K}$ 。 Li. Entre ellos, principalmente el $\mathrm{NaOH}$, el $\mathrm{Na}_{2} \mathrm{CO}_{3}$ y silicato sódico (waterglass) son los activadores alcainos más efectivos desde el punto de vista mecánico y de otras propiedades.

Algunos autores (9) han indicado que la mezcla de activadores también tiene un carácter positivo sobre el desarrollo de resistencias. Concretamente, la mezcla $\mathrm{Na}_{2} \mathrm{CO}_{3}+\mathrm{Na}_{2} \mathrm{SO}_{4}+$ cal hidratada ha resultado ser tan efectiva como la dosificación más óptima de $\mathrm{NaOH}$.

Las funciones de las sustancias activadoras deben de ser las siguientes:

i) Acelerar la solubilización de la escoria.

ii) Favorecer la formación de hidratos estables de baja solubilidad. 
iii) Favorecer la formación de una estructura compacta con esos hidratos formados.

Únicamente cuando el $\mathrm{pH}$ de las disoluciones de las sustancias activadoras es superior a 12 se consigue, no sólo la solvatación de la escoria, sino también la formación de compuestos hidratados estables. A pH ácidos, aunque también sea posible la disolución de la escoria (ver Fig. 1), los hidratos no son estables y no es posible la formación de una estructura coherente de los mismos.

Las disoluciones saturadas de $\mathrm{CaSO}_{4} \cdot 2 \mathrm{H}_{2} \mathrm{O}$ ó el $\mathrm{Na}_{2} \mathrm{SO}_{4}$ tienen $\mathrm{pH}$ de 10,6 y 7 , respectivamente. A esos $\mathrm{pH}$, la cantidad de sílice soluble procedente de la escoria es muy pequeña, tal y como puede observarse en la Fig. 1; es decir, ambos compuestos no producen una activación importante en la escoria. Sin embargo, si dichos sulfatos se encuentran en un medio más básico, la hidratación de la escoria podría verse notablemente acelerada, por la disolución inicial de la escoria y posterior formación de ettringita. La formación de este hidrato hace que la concentración de iones $\mathrm{Ca}^{2+}$ y $\mathrm{Al}^{3+}$ en la fase líquida descienda, lo que favorece que se siga produciendo la disolución de la escoria.

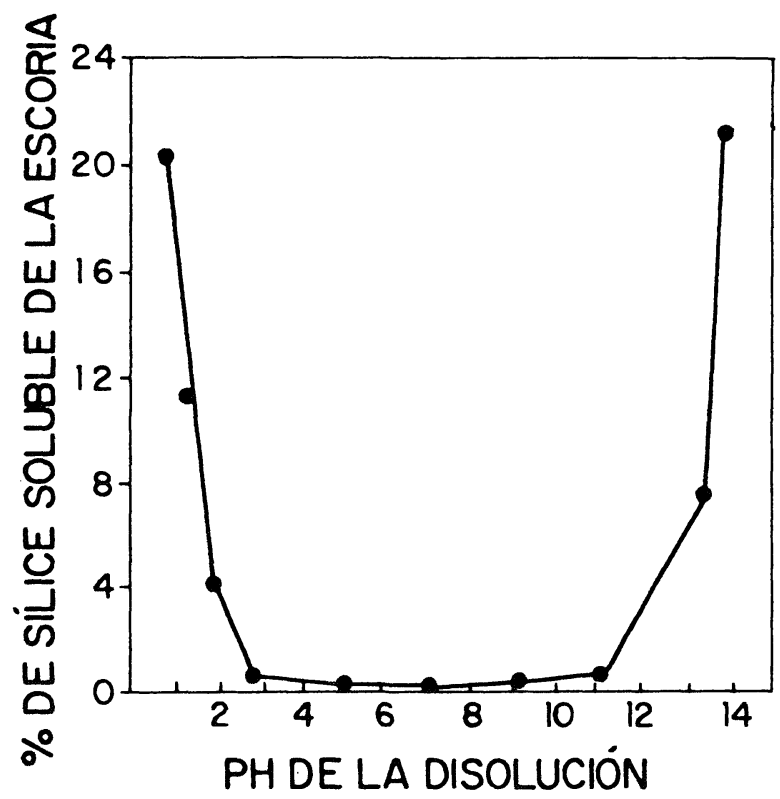

Fig. 1.- Cantidad de sílice soluble de la escoria en función del $\mathrm{pH}$ de la disolución activadora (15).

\section{MECANISMOS DE ACTIVACIÓN ALCALINA DE LAS ESCORIAS}

La hidratación de la escoria por activación alcalina se puede definir como un proceso complejo en el que inicialmente se produce la destrucción de la estructura de la escoria $y$, posteriormente, una policondensacióncristalización de los productos de hidratación formados.

Zhou Huanhai et al (22) al estudiar el mecanismo de hidratación de una escoria granulada de alto horno con silicato sódico, comprueban que, pese a ser los procesos de hidratación de las escorias activadasy del cemento portland ordinario muy diferentes, sus curvas calorimétricas son similares. Proponen que el mecanismo de activación alcalina de las escorias se puede clasificar en cinco etapas: inicial, inducción, aceleración, deceleracióny finalización.

La etapa inicial de laactivación corresponde a la reac-ción entre los iones silicato del $\mathrm{Na}_{2} \mathrm{SiO}_{3}$ y los iones $\mathrm{Ca}^{2+}$ disueltos procedentes de la escoria, para formar un hidrosilicato cálcico tipo CSH. La hidratación se para cuando los granos de escoria quedan recubiertos del hidrato $\mathrm{CSH}$, iniciándose la etapa denominada de inducción.

En tiempo de duración de la etapa de inducción va a depender del pH de la solución en contacto con las fases sólidas. Cuanto mayor sea el $\mathrm{pH}$ menor será el período de inducción. La finalización de esta etapa de inducción coincide con el inicio de la etapa de aceleración en la hidratación de la escoria. En esta etapa se produce la reacción entre los aluminatos y silicatos con los iones metálicos $(\mathrm{Na}, \mathrm{Ca}, \mathrm{Mg}$, etc.) para formar un "CSH secundario" y otros productos de hidratación de naturaleza más compleja.

La transición de la etapa de inducción a la de aceleración se puede explicar de acuerdo a la teoría de la nucleación. Cuando la concentración de los iones $\mathrm{Ca}^{2+}$ y $\mathrm{SiO}_{4}^{4-}$ procedentes de la disolución de la escoria alcanzan la concentración de saturación del silicato cálcico hidratado, se produce la nucleación, crecimiento y precipitación del citado hidrato. El proceso de hidratación continúa, pero gradualmente va disminuyendo la velocidad dela reacción. Esto se atribuye a una precipitación masiva de productos de reacción alrededor de los granos de escoria, y a una disminución del agua libre, lo cual conduce a impedir o dificultar los procesos de difusión, y, por lo tanto, disminuir progresivamente las reacciones de hidratación. Estas etapas corresponden con las de deceleración y finalización.

A. Fernández-Jiménez et al (23) han encontrado que la duración del período de inducción depende de la naturaleza de la sustancia activadora. Así, disoluciones de diluidas de $\mathrm{Ca}(\mathrm{OH})_{2}$ y yeso actúan como retardadores del período de inducción, mientras que disoluciones de $\mathrm{NaOH} 1 \mathrm{~N}$ y $\mathrm{Na}_{2} \mathrm{CO}_{3} 2 \mathrm{~N}$ actúan como aceleradores.

Wang y Scrivener (24), al estudiar el desarrollo microestructural de las pastas de escorias activadas alcalinamente, concluyen que, en las primeras edades, el 
mecanismo de la hidratación transcurre a través de la disolución de la escoria y la precipitación de hidratos. A edades, más avanzadas, la presencia de anillos de productos de reacción alrededor de los granos de escoria, sugieren que la hidratación debe proseguir por un mecanismo de reacción en estado sólido.

El papel jugado por el catión alcalino en el proceso de hidratación de la escoria es objeto de controversia por los distintos autores. Para la mayoría de los investigadores (2, 3,25 ), el ión alcalino participa en el intercambio catiónico y actúa como un catalizador de la reacción en las primeras edades, de acuerdo al siguiente esquema:

$$
\begin{aligned}
& \text { Si-O }+\mathrm{R}^{+}=\text {Si-O-R } \\
& \text { Si-O-R }+\mathrm{OH}^{-}=\mathrm{Si}-\mathrm{O}-\mathrm{R}-\mathrm{OH}^{-} \\
& \text {Si-O-R-OH }
\end{aligned}
$$

En opinión de esos mismos autores, a edades más avanzadas en la hidratación, ese catión alcalino participa activamente en la formación de fases tipo zeolitas-alcalinas.

Para otros autores $(12,26)$ esos cationes alcalinos únicamente tienen el papel de compensar por balanceo las cargas negativas de los aniones y los $\mathrm{OH}^{-}$.

Es dificil determinar con exactitud la situación de esos iones alcalinos en las pastas hidratadas de escorias activadas. Una de las maneras es someter a las pastas a un proceso de lavado con agua y cuantificar la concentración de $\mathrm{Na}$ o K disuelto. Por diferencia se puede establecer el álcali fijado. Procesos de lavado muy rigurosos pueden extraer el álcali estructuralmente unido en disolución sólida en el CSH (27). A través de las técnicas microanalíticas es difícil distinguir entre el álcali adsorbido o el estructuralmente incorporado a los productos de hidratación. Taylor (28) indica que la cantidad de álcali (ya sea $\mathrm{Na}$ o K) que pueden encontrarse en disolución sólida en la estructura del CSH es relativamente pequeña, concretamente relaciones $\mathrm{Na} / \mathrm{Ca}$ y $\mathrm{K} / \mathrm{Ca}$ de 0,01 . La mayor parte de los iones alcalinos en una pasta de cemento portland madura se encuentran en la fase acuosa de los poros de la pasta.

Shi et al (5) destacan el papel jugado por el anión del activador. Indican que el efecto activador no depende del pH original de la solución sino de la naturale- za del anión o grupo de aniones del activador utilizado. Es decir, habria un carácter selectivo de los activadores.

De cualquier manera, los estudios en torno a los mecanismos de hidratación de los cementos de escorias activadas alcalinamente son difíciles de llevar a cabo. Ello es debido, por una parte, a la elevada complejidad de algunas de las disoluciones activadoras, comoel waterglass, ya que éste puede encontrarse en muy diferentes formas (diferentes grados de polimerización, distinto $\mathrm{n}^{\circ}$ de moléculas de agua de constitución, etc.); y por otra, a causa de la complejidad de los productos de hidratación amorfos formados, cuya grado de polimerización, cristalinidad, contenido y variedad es muy difícil de controlar (4).

\section{PRODUCTOS DE HIDRATACIÓN}

La naturaleza de los productos de hidratación, formados como consecuencia de la activación alcalina de escorias, es uno de los aspectos más controvertidos y que más estudios y dedicación precisan.

En lo único en lo que parecen coincidir los distintos autores es en considerar al silicato cálcico hidratado $\mathrm{CSH}$ (B) como el principal producto de hidratación. Esta fase gel se diferencia de la de una pasta de cemento portland por una menor relación $\mathrm{C} / \mathrm{S}$. La formación de otras fases o compuestos hidratados va a depender del tipo y cantidad del activador utilizado, de la estructura y composición de la escoria y las condiciones de curado bajo las cuales se va a producir el endurecimiento (8).

Según Glukhovsky et al (2), aparte del gel CSH, también se pueden encontrar en una pasta de cemento de escorias activadas: hidronefelinas $\left(\mathrm{R}_{2} \mathrm{O} \cdot \mathrm{Al}_{2} \mathrm{O}_{3} \cdot 2 \mathrm{SiO}_{2} \cdot \mathrm{nH}_{2} \mathrm{O}\right)$, natrolita $\left(\mathrm{R}_{2} \mathrm{O} \cdot \mathrm{Al}_{2} \mathrm{O}_{3} \cdot 3 \mathrm{SiO}_{2} \cdot \mathrm{nH}_{2} \mathrm{O}\right)$, analcita $\left(\mathrm{R}_{2} \mathrm{OAl}_{2} \mathrm{O}_{3} \cdot 4 \mathrm{SiO}_{2} \cdot \mathrm{nH}_{2} \mathrm{O}\right)$, moscovita y paragonita $\left(\mathrm{R}_{2} \mathrm{O} .3 \mathrm{Al}_{2} \mathrm{O}_{3} .6 \mathrm{SiO}_{2} 2 . \mathrm{n} \mathrm{H}_{2} \mathrm{O}\right)$, g i s mondita $\left(\mathrm{CaOAl}_{2} \mathrm{O}_{3} \cdot 2 \mathrm{SiO}_{2} \cdot \mathrm{nH}_{2} \mathrm{O}\right)$, hidrogranates y una mezcla de hidroaluminosilicatos de potasio-calcio y sodio-calcio. Por el contrario, no presentan ni $\mathrm{Ca}(\mathrm{OH})_{2}$ ni aluminatos cálcicos hidratados ni sulfoaluminatos cálcicos hidratados, típicos de una pasta de cemento portland.

La solubilidad de todos esos compuestos es menor que la de los compuestos hidratados de una pasta de cemento portland, tal y como puede observarse en la Tabla I (25). Esa menor solubilidad esta indicando una mayor estabilidad química de las pastas de cementos de escorias activadas alcalinamente frente a las pastas de cemento portland.

A. Fernández-Jiménez (29) encuentra al activar una escoria de alto horno española con $\mathrm{NaOH}$, la formación de un gel CSH semicristalino con espaciados en 12,50, 2,80 y 1,83 $\AA$. A través de espectroscopía IR se confirmaba la mayor ordenación estructural por las bandas agudas situadas en 970 y $454 \mathrm{~cm}^{-1}$. Wang y Scrivener (24), al estudiar escorias activadas con $\mathrm{NaOH}$ obtienen resultados similares.

Algunos autores $(24,29,30)$ han indicado la formación de una fase tipo hidrotalcita $\left(\mathrm{Mg}_{6} \mathrm{Al}_{2} \mathrm{CO}_{3}(\mathrm{OH})_{16} .4 \mathrm{H}_{2} \mathrm{O}\right)$ en las pastas de escorias activadas con $\mathrm{NaOH}$ y en cementos 
Solubilidad de los Productos de Hidratación de las Escorias Activadas Alcalinamente y de los Cementos Portland (25)

\begin{tabular}{|c|c|c|}
\hline MINERAL & FÓRIMULA ESTEQUIOMÉTRICA & SOLUBILIDAD $\mathrm{kg} / \mathrm{m}^{3}$ \\
\hline \multicolumn{3}{|c|}{ Cemento de Escoria Activada } \\
\hline $\begin{array}{l}\mathrm{CSH}(\mathrm{B}) \\
\text { Xonotlita } \\
\text { Riversidita } \\
\text { Plombierita } \\
\text { Gyrolita } \\
\text { Calcita } \\
\text { Hidrogranate } \\
\text { Hidrosilicato de sodio y cálcio } \\
\text { Thomsonita } \\
\text { Hidronephelina } \\
\text { Natrolita } \\
\text { Analcima }\end{array}$ & $\begin{array}{l}5 \mathrm{CaO} \cdot 6 \mathrm{SiO}_{2} \cdot \mathrm{nH}_{2} \mathrm{O} \\
6 \mathrm{CaO} \cdot 6 \mathrm{SiO}_{2} \cdot \mathrm{H}_{2} \mathrm{O} \\
5 \mathrm{CaO} \cdot 6 \mathrm{SiO}_{2} \cdot 3 \mathrm{H}_{2} \mathrm{O} \\
5 \mathrm{CaO} \cdot 6 \mathrm{SiO}_{2} \cdot 10 \cdot 5 \mathrm{H}_{2} \mathrm{O} \\
2 \mathrm{CaO} \cdot 3 \mathrm{SiO}_{2} \cdot 2 \cdot 5 \mathrm{H}_{2} \mathrm{O} \\
\mathrm{CaCO} \\
3 \mathrm{CaO} \cdot \mathrm{Al}_{2} \mathrm{O}_{3} \cdot 1 \cdot 5 \mathrm{SiO}_{2} \cdot 3 \mathrm{H}_{2} \mathrm{O} \\
(\mathrm{Na}, \mathrm{Ca}) \mathrm{SiO}_{4} \cdot \mathrm{nH}_{2} \mathrm{O} \\
(\mathrm{Na}, \mathrm{Ca}) \mathrm{Al}_{2} \mathrm{O}_{3} \cdot \mathrm{Si}_{2} \mathrm{O}_{5} \cdot 6 \mathrm{H}_{2} \mathrm{O} \\
\mathrm{Na}_{2} \mathrm{O} \cdot \mathrm{Al}_{2} \mathrm{O}_{3} \cdot 2 \mathrm{SiO}_{2} \cdot 2 \mathrm{H}_{2} \mathrm{O} \\
\mathrm{Na}_{2} \mathrm{O} \cdot \mathrm{Al}_{2} \mathrm{O}_{3} \cdot 3 \mathrm{SiO}_{2} \cdot 2 \mathrm{H}_{2} \mathrm{O} \\
\mathrm{Na}_{2} \mathrm{O} \cdot \mathrm{Al}_{2} \mathrm{O}_{3} \cdot 4 \mathrm{SiO}_{2} \cdot 2 \mathrm{H}_{2} \mathrm{O}\end{array}$ & $\begin{array}{l}0,050 \\
0,035 \\
0,050 \\
0,050 \\
0,051 \\
0,014 \\
0,020 \\
0,050 \\
0,050 \\
0,020 \\
0,020 \\
0,020 \\
\end{array}$ \\
\hline \multicolumn{3}{|c|}{ Cemento Portland } \\
\hline $\begin{array}{l}\text { Hidróxido de cálcio } \\
\mathrm{C}_{2} \mathrm{SH}_{2} \\
\mathrm{CSH}_{(\mathrm{B})} \\
\text { Hidroaluminato tetracálcico } \\
\text { Hidroaluminato tricálcico } \\
\text { Ettringita }\end{array}$ & $\begin{array}{l}\mathrm{Ca}(\mathrm{OH})_{2} \\
2 \mathrm{CaO} \cdot \mathrm{SiO}_{2} \cdot \mathrm{nH}_{2} \mathrm{O} \\
5 \mathrm{CaO} \cdot \mathrm{SiO}_{2} \cdot \mathrm{nH}_{2} \mathrm{O} \\
4 \mathrm{CaO} \cdot \mathrm{Al}_{2} \mathrm{O}_{3} \cdot 13 \mathrm{H}_{2} \mathrm{O} \\
3 \mathrm{CaO} \cdot \mathrm{Al}_{2} \mathrm{O}_{3} \cdot 6 \mathrm{H}_{2} \mathrm{O} \\
3 \mathrm{CaO} \cdot \mathrm{Al}_{2} \mathrm{O}_{3} \cdot 3 \mathrm{CaSO}_{4} \cdot 31 \mathrm{H}_{2} \mathrm{O}\end{array}$ & $\begin{array}{l}1,300 \\
1,400 \\
0,050 \\
1,080 \\
0,560 \\
1,754\end{array}$ \\
\hline
\end{tabular}

portland y de escorias. $\mathrm{C}_{4} \mathrm{AH}_{13}$ se forma en escorias activadas con $\mathrm{NaOH}$, y ha sido identificada por unas placas de $0,1-0,2 \mu \mathrm{m}$ de espesor y de aproximadamente $12 \mu \mathrm{m}$ de diámetro (24). Otros autores (29) han observado la presencia de fases carbonatadas tipo $\mathrm{C}_{4} \mathrm{AcH}_{11} \mathrm{y} \mathrm{C}_{8} \mathrm{~A}_{2} \mathrm{cH}_{24}$ en las pastas de escorias activadas con $\mathrm{NaOH}$ y $\mathrm{Ca}(\mathrm{OH})_{2}$.

Kutti (8), al estudiar los productos de hidratación de una escoria de alto horno sueca activada con $\mathrm{NaOH}$, encuentra que la pasta está constituida mayoritariamente por dos compuestos: uno es una fase tipo CSH y el otro es un gel rico en sílice. Este último gel es el que controla la retracción, la permeabilidad y las resistencias de los cementos, morteros y hormigones elaborados con esas escorias activadas. Más adelante se discutirá el papel de ese gel de sílice con las propiedades del material. La formación de ese gel rico en sílice también se ha encontrado en pastas procedentes de la activación con $\mathrm{Na}_{2} \mathrm{SiO}_{3}(4)$.

Las escorias activadas con $\mathrm{Na}_{2} \mathrm{SiO}_{3}$ contienen menor cantidad de sales de silicoaluminatos que aquéllas activadas con $\mathrm{Na}_{2} \mathrm{CO}_{3}$. Wang et al (4) indican que ello es debido a la formación de un gel de sílice procedente de la hidrólisis del $\mathrm{Na}_{2} \mathrm{SiO}_{3}$, el cual puede retardar la disolución del $\mathrm{Al}_{2} \mathrm{O}_{3}$ de la escoria.

\section{CARACTERÍSTICAS Y PROPIEDADES DE LOS CEMENTOS DE ESCORIAS ACTIVADAS ALCALINAMENTE}

Los cementos y hormigones de escorias activadas alcalinamente se caracterizan por presentar las siguientes ventajas respecto a los correspondientes de cemento portland ordinario:

* Más rápidas y mayores resistencias mecánicas.

* Menor calor de hidratación.

* Mejor impermeabilidad.

* Mejor comportamiento frente a la carbonatación.

* Mayor resistencia al hielo-deshielo.

* Mayor resistencia a altas temperaturas.

* Mayor resistencia al ataque químico.

* Mayor resistencia de la interfase árido-matriz.

* Mayor protección de las armaduras metálicas.

* Resistencia bacteriológica y biocida.

* Menor costo y mayor ahorro de energía. 
Entre las desventajas hay que mencionar:

* Fraguado más rápido.

* Mayor retracción y formación de microfisuras.

* Posibilidad de que se produzca mayor reacción áridoálcali.

* Mayor formación de eflorescencias.

A continuación se pasa a describir el estado del conocimiento en torno a cada una de estas características y propiedades.

\section{Resistencias Mecánicas}

En morteros de escorias activadas alcalinamente se pueden alcanzar resistencias de 40-120 MPA (2). En hormigones estos valores pueden llegar a ser de 60-150 MPA, tanto con áridos calizos como silíceos. Se pueden alcanzar resistencias de hasta 30-35 MPA a un sólo día, 40-55 MPA a los dos días y de 65-75 MPA a los tres días de hidratación. Estas resistencias a edades tempranas son muy superiores a las que desarrollan los cementos portland de altas resistencias, los cementos de escorias e, incluso, los cementos aluminosos (3).

A lo largo del tiempo se produce un incremento de esas resistencias. Alcabo de 10 añosde servicios, las resistencias pueden llegar a ser un $100-200 \%$ superiores a las que desarrolla- ron a los 28 días (2).

Las resistencias mecánicas de los hormigones de escorias activadas tienen una desviación mayor que las resistencias de los hormigones de cemento portland. Ello es debido a que el desarrollo de resistencias de esos hormigones depende tanto de la naturaleza de la escoria como del agente activante.

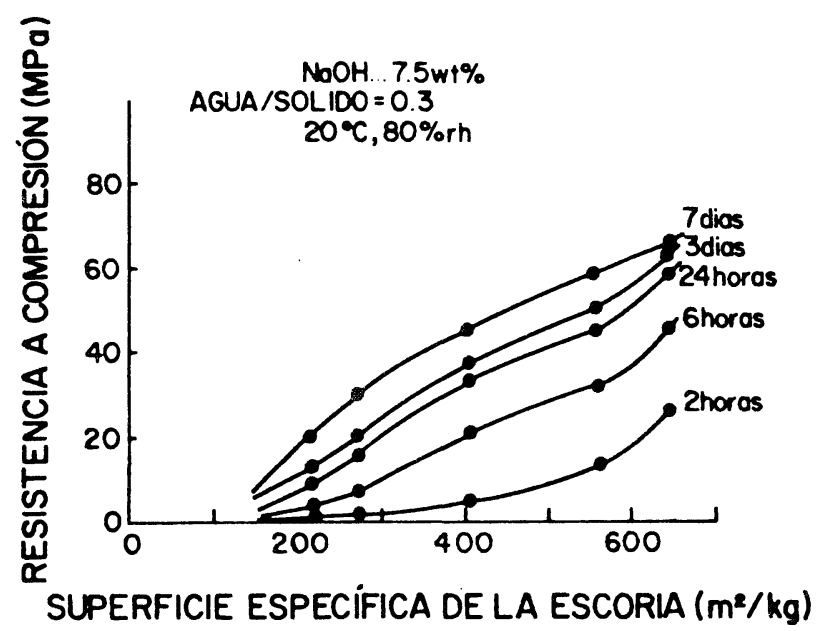

Fig. 2.- Influencia de la superficie específica de la escoria en el desarrollo de resistencias mecánicas (10).
Según Wang et al (20) los factores que afectan a las resistencias mecánicas de las escorias activadas alcalinamente son: el tipo de activador, el método de adición del activador, la dosificación del activador, la naturaleza y finura de la escoria, la temperatura de curado, las relaciones agua/escoria y los aditivos.

De los resultados obtenidos en sus trabajos indican que el waterglass ó silicato sódico es el mejor activador en todos los casos.

El grado de polimerización de los aniones silicato tiene una gran influencia sobre las resistencias mecánicas del material. Las escorias activadas con $\mathrm{Na}_{2} \mathrm{SiO}_{3}$ desarrollan mayores resistencias mecánicas que las activadas con $\mathrm{NaOH}$. La interpretación que de ello hacen algunos autores (4) es que, aunque la reactividad de la escoria es la misma, el grado de polimerización de los aniones silicato es muy superior en las pastas de escorias activadas con $\mathrm{Na}_{2} \mathrm{SiO}_{3}$.

Respecto al método de adicionar el componente alcalino a la mezcla, concluyen que la forma de disolución es el mejor sistema en todos los casos. La proporción de óptima de $\mathrm{Na}_{2} \mathrm{O}$ debe de encontrarse entre $3,0-5,5 \%$ en peso de escoria. El rango de superficie específica óptimo de la escoria debe de oscilar entre $4.000-5.500 \mathrm{~cm}^{2} / \mathrm{g}$. La relación más idónea de disolución (volumen)/escoria (peso) debe de encontrarse entre $0,38-0,45$.

En las figuras 2 y 3 se muestra el efecto de la superficie específica de la escoria y de la proporción de $\mathrm{NaOH}$ sobre el desarrollo de resistencias mecánicas en probetas de pasta de cemento (10). En este estudio los autores comprobaron que el contenido óptimo de $\mathrm{NaOH}$ para alcanzar las máximas resistencias oscilaba entre 7,5 y $10,0 \%$ en peso.

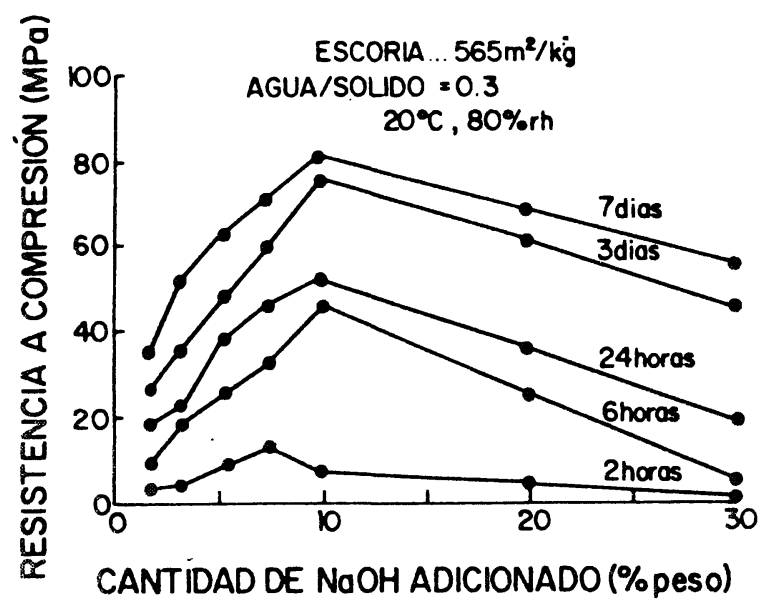

Fig. 3.- Influencia de la cantidad de $\mathrm{NaOH}$ adicionado en el desarrollo de resistencias mecánicas (10). 
$\mathrm{Al}$ aumentar la temperatura de curado se incrementan las resistencias mecánicas de los cementos de escorias activadas $(20,31)$. Como consecuencia de la temperatura, se forman en la activación alcalina de escorias productos de hidratación cristalinos, mientras que a temperatura ambiente esos productos son mayoritariamente de naturaleza amorfa.

Según Wang et al (20) el efecto positivo desde el punto de vista resistente del incremento de la temperatura de curado se manifiesta más marcadamente en las escorias ácidas ó neutras, y sobre activadores de baja ó media alcalinidad. Shi et al (33) encontraron que al activar alcalinamente escorias de alto horno y fosforosas a $150^{\circ} \mathrm{C}$, se obtenían pastas de elevadas resistencias con unos poros de radio comprendidos entre 10 y $100 \AA$; en iguales condiciones las pastas de cemento portland tienen poros de radio muy superior a $100 \AA$.

Sin embargo, aún no está completamente establecida la relación existente entre el comportamiento resistente del material final y el tiempo y temperatura de curado y otros factores, tales como tipo y superficie específica de la escoria y naturaleza y concentración del agente activante.

\section{Calor de Hidratación}

Los cementos de escorias activadas alcalinamente se caracterizan por bajos calores de hidratación. Ello se justifica por la menor energía de hidratación de los metales alcalinos [K- 3,43.10 ${ }^{8}, \mathrm{Na}-4,14.10^{8} \mathrm{~J} /(\mathrm{kg}$-ión)] frente a la de los metales alcalinotérreos $\left[\mathrm{Ca}-15,11.10^{8} \mathrm{~J} /\right.$ (kg-ión)] (3). El calor de hidratación de los cementos de escorias activadas alcalinamente es 1/3-1/2 menor que el desarrollado por los cementos portland. Esos bajos calores de hidratación permiten el empleo de esos cementos de escorias activadas para la fabricación de grandes obras en masa, como por ejemplo, presas.

\section{Impermeabilidad}

Los morteros y hormigones de escorias activadas alcalinamente presentan una impermeabilidad superior que los elaborados con cementos portland. La velocidad de difusión de los iones $\mathrm{Cl}^{-}$en pastas de cemento de escorias activadas es de 30 a 40 veces más lenta que en las pastas de cemento portland, a iguales relaciones agua/ligante (32). Esto está muy relacionado con la mayor protección de las armaduras embebidas en los hormigones elaborados con este tipo de cementos.

\section{Comportamiento frente a la Carbonatación}

Byfors et al (32) han comprobado que F-hormigones (hormigones de escorias activadas con $2,75 \% \mathrm{NaOH}$ y
$1,0 \% \mathrm{Na}_{2} \mathrm{CO}_{3}$ ) con relaciones agua/ligante de 0,35 tienen iguales velocidades de carbonatación que aquellos elaborados con cemento portland y relación agua/cemento de 0,6 . Algunos autores (24) han encontrado una relación muy estrecha entre las resistencias mecánicas de los hormigones de escorias activadas y su velocidad a la carbonatación. Así, hormigones de resistencias inferiores a $30 \mathrm{MPA}$, tienen una rápida velocidad de carbonatación, cuando las resistencia se encuentra entre 30-50 MPA tienen una velocidad media de carbonatación, $y$, finalmente, cuando las resistencias de los hormigones son superiores a los 50MPA, la velocidad y grado de carbonatación es muy inferior a la de los correspondientes hormigones de cemento portland. Deja y Malolepszy (33) encontraron que después de 90 días de conservación en atmósfera de $\mathrm{CO}_{2}$, los hormigones de escorias activadas habian experimentadoun incremento de un $50 \%$ de sus resistencias mecánicas a compresión. Los autores lo interpretaban como consecuencia de la formación de una estructura muy compacta (con una disminución de la porosidad) entre el $\mathrm{CaCO}_{3}$ y el C-S-H. Los estudios microestructurales realizados han demostrado que el $\mathrm{CaCO}_{3}$ se encontraba rellenando espacios vacíos y fisuras. En este estudio, los citados autores concluyeron que la velocidad de carbonatación era menor cuando el activador era el silicato sódico.

\section{Resistencia a Bajas y Altas Temperaturas}

La mayor resistencia de los cementos y hormigones de escorias activadas alcalinamente al frio se debe a que las temperaturas de congelación de las disoluciones acuosas de metales alcalinos están por debajo de $0^{\circ} \mathrm{C}$ (del orden de $-15^{\circ} \mathrm{a}-20^{\circ} \mathrm{C}$ ). Esto hace que dichos hormigones pueden permanecer estables, sin sufrir destrucciones por expansiones, debidas a la congelación, hasta temperaturas del orden de $-30^{\circ} \mathrm{C}$. En estudios realizados se ha comprobado que estos hormigones son capaces de superar los 1.000 ciclos al hielo-deshielo (2). Son, pues, hormigones muy adecuados para climas fríos.

No sólo son resistentes al frio sino también a las altas temperaturas. Estos cementos de escorias activadas alcalinamente son capaces de resistir, sin perder sus características estructurales, hasta temperaturas de $700^{\circ} \mathrm{C}$ (24), mientras que los cementos portland pierden esas características por debajo de los $500^{\circ} \mathrm{C}$. Lo que hace que esos cementos $u$ hormigones puedan ser utilizados como materiales refractarios.

\section{Resistencia al Ataque Químico}

Los cementos y hormigones de escorias activadas alcalinamente son más estables frente a medios agresivos de sulfatos, agua de mar y medios ácidos, que los de 
cemento portland $(2,32)$. Después de exposiciones de 1.340 días, Byfors et al (32) concluyeron que morteros de cemento $\mathrm{F}$ eran resistentes a disoluciones saturadas de

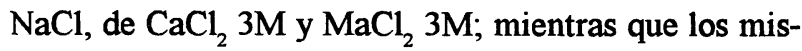
mos morteros presentaban fisuras cuando se encontraban parcialmente sumergidos en agua destilada. Wang (4) indica que hormigones de escorias activadas incrementan su resitencias después de permenecer sumergidos en disoluciones de agua de mar y $\mathrm{MgSO}_{4}$, mientras que los hormigones de portland se destruyen, en igualdad de condiciones, a los 6 meses de ensayo.

\section{Resistencia de la Interfase Árido-Matriz}

Casi todos los autores $(3,24,4)$ coinciden en afirmar que la interfase árido-pasta en los hormigones de escorias activadas alcalinamente presenta una elevada cohesión y baja porosidad.

En los hormigones de cemento portland, la zona interfacial árido-pasta de cemento corresponde con el punto débil del citado hormigón. Esa zona interfacial se caracteriza por presentar una elevada porosidad y cristales grandes y orientados de productos de hidratación, concretamente $\mathrm{Ca}(\mathrm{OH})_{2}$ y AFt. En los hormigones de escorias activadas alcalinamente no hay cristales grandes de $\mathrm{Ca}(\mathrm{OH})_{2}$ ni de Aft, sino que por el contrario, en la zona interfacial, debido a la presencia de álcalis, se puede producir una interacción química entre dichos álcalis y los materiales arcillosos de la superficie de los áridos, con la formación de unas fases de naturaleza cementícia que conducen a un incremento muy importante de la adherencia y resistencia

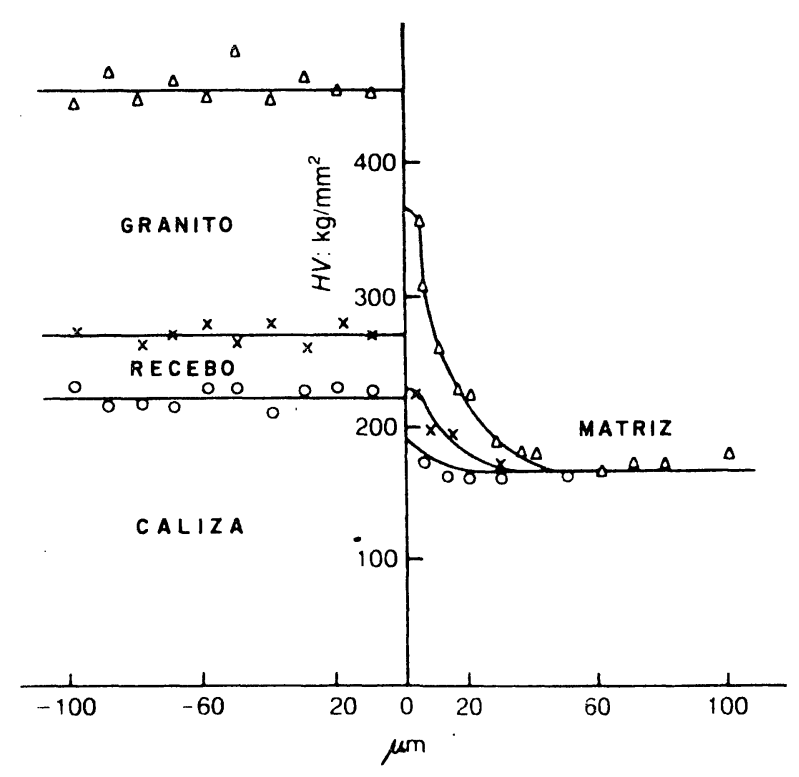

Fig. 4.- Propagación de las fisuras en la interfase árido-matriz en hormigones de escorias activadas alcalinamente (4). de la interfase; haciéndo que, en muchas ocasiones, esa interfase sea más resistente que la propia matriz. Esto haque que la fisura de fractura progrese, casi siempre, a través del árido, ya sea este silíceo o calcáreo (veáse figura 4 (4)).

\section{Protección de las Armaduras Metálicas}

La fase acuosa de los hormigones de escorias activadas alcalinamente tiene un $\mathrm{pH}$ de 11,5-12. Esta elevada alcalinidad asegura un medio protector de las armaduras metálicas. La pérdida de masa de las armaduras de acero demuestra que la corrosión en estos hormigones de escoria es aproximadamente de 2-2,5 veces menor que en el correspondiente de cemento portland. Adicionalmente, y como ya se ha comentado, la impermeabilidad de los hormigones es muy superior, y el transporte de iones agresivos como los iones $\mathrm{Cl}^{-}$en más difícil, con velocidades de difusión menores que en los hormigones de cemento portland.

\section{Resistencia al Biodeterioro}

Los hormigones de escorias activadas alcalinamente tienen una resistencia notable al crecimiento de bacterias, hongos y algas, así como una elevada resistencia hidrodinámica (3). Estas características hace que estos hormigones sean especialmente interesantes parasu empleo en el almacenamiento de agua potable.

\section{Ahorro Energético y Económico}

La fabricación de hormigones de escorias activadas alcalinamente con elevadas resistencias iniciales es mucho más económico que la elaboración de hormigones de altas resistencias de cemento portland. Según Glukhovshij et al (3), el ahorro en la fabricación de materiales de escorias activadas alcalinamente se debe, principalmente, a la dismunución en el consumo de un $70 \%$ en combustible y alrededor de un $60-70 \%$ en energía eléctrica.

\section{Fraguado Rápido}

Algunos cementos de escorias activadas pueden empezar a fraguar a los 15 minutos de hidratación (27). Diferentes aditivos como boratos y fosfatos han sido utilizados con éxito como retardadores del fraguado en cementos de escorias activadas de resistencia media (34), sin embargo, no son útiles en cementos de escorias activadas de resistencia elevada.

Algunos estudios han demostrado que la cal apagada o la cal hidráulica pueden controlar los tiempos de fraguado de esos cementos, cuando se emplea como activador silicato sódico, de módulo 2,85 (35). 
Los procesos y mecanismos de fraguado de los cementos de escorias activadas alcalinamente no son suficientemente conocidos. Ello hace que, hasta la fecha, sea difícil proponer soluciones. En cualquier caso, éste es un aspecto que requiere nuevas y profundas investigaciones.

\section{Retracción al Secado y Formación de Microfisuras}

Las pastas de cemento de escorias activadas alcalinamente con $\mathrm{NaOH}$ y waterglass, experimentan una retracción por secado muy superior a la que ocurre en las pastas de cemento portland. Esa mayor contracción de volumen provoca la formación de una elevada microfisuración en las correspondientes pastas.

Según Wang et al (27) los factores que afectan a la retracción por secado de los hormigones de escorias activadas son: la superficie específica de la escoria, la relación disolución alcalina/escoria, la relación árido/ escoria, el módulo de waterglass y la temperatura de curado.

Superficies específicas altas de la escoria, bajas relaciones disolución activadora/escoria, bajos módulos de waterglass y altas relaciones árido/escoria, parecen favorecen retracciones controladas.

Kutti(8)interpretaque esa retracción se debe a laformación, principalmente, de dos productos de hidratación en las pastas de escorias activadas. Uno de esos productos es una fase tipo gel CSH y el otro es una fase gel rica en sílice y con propiedades similares a las de la sílice gel. En la figura 5 se muestra un esquema de la disposición de esas dos fases en una pasta de escoria activada (8).
La formación y propiedades de ese gel CSH es presumiblemente similar a la del gel CSH de la pasta del cemento portland. Es decir, la fase es relativamente estable frente al secado. Por otra parte, el gel de sílice se forma en las etapas iniciales de la hidratación y contiene una elevada cantidad de agua. Este agua no está químicamente combinada y su eliminación es relativamente fácil. Durante el secado, ese agua se elimina y la fase gel de sílice experimenta una contracción de volumen que provoca fisuración dentro del propio gel. Así pues, en opinión de este autor, el responsable de la retracción y fisuración de las pastas y hormigones de escorias activadas es ese gel de sílice.

Al igual que en el apartado anterior relativo al fraguado rápido, la retracción por secado de este tipo de cementos requiere mayores esfuerzos e investigaciones.

\section{Reacción Árido-Álcali}

Como es sabido, la escoria de alto horno es una adición efectiva para minimizar o impedir la reacción árido-álcali en los hormigones de cemento portland.

Según algunos autores $(25,35)$, no es posible que se produzca la reacción árido-álcali en los hormigones de escorias activadas, ya que todos los álcalis se encuentran combinados formando los distintos productos de hidratación. Sin embargo, y como ya se ha indicado, la extensión a la cual se encuentran ligados esos álcalis en los distintos productos de hidratación no es,aún, suficientemente conocida.

Algunos autores (7) han encontrado expansiones en morteros de escorias activadas que atribuyen a la formación
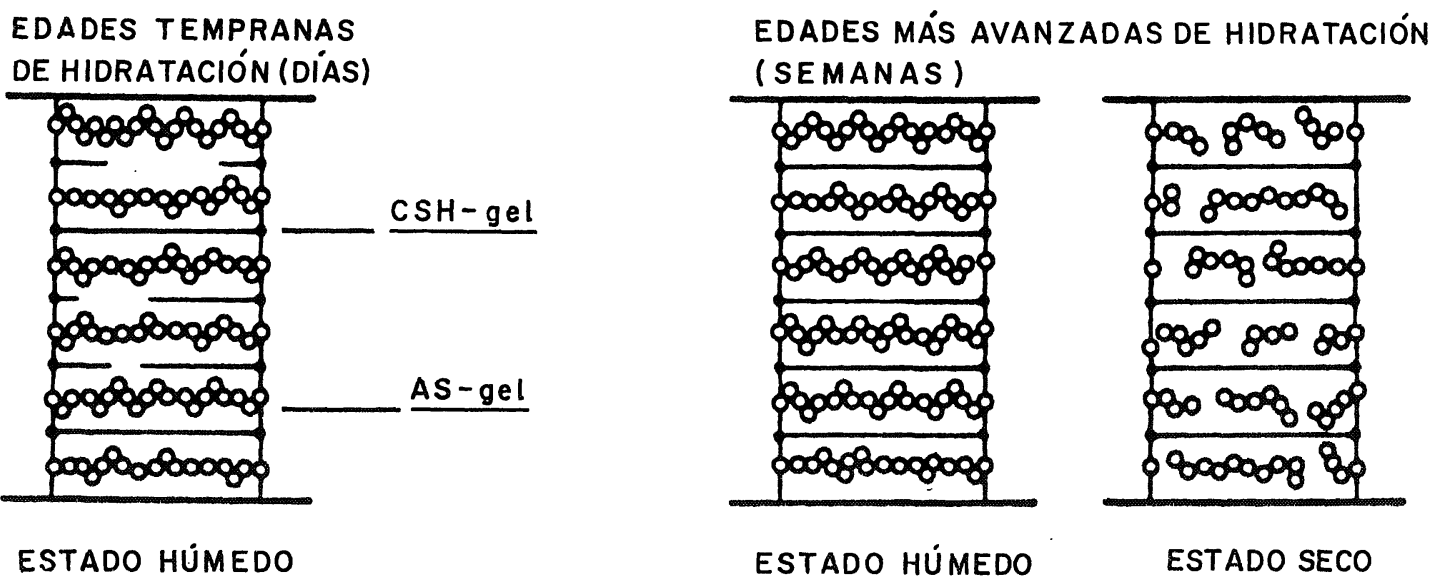

Fig. 5.- Modelo microestructural de las pastas de escorias activadas a diferentes edades de hidratación (8). 
de productos expansivos de reacción árido-álcali.

Como se desprende de lo anteriormente expuesto, es preciso profundizar en el conocimiento y comportamiento de estos materiales frentea áridos potencialmente reactivos.

\section{Formación de Eflorescencias}

En los morteros y hormigones de escorias activadas alcalinamente se forman una mayor cantidad de eflorescencias. Esas eflorescencias pueden ser de diferente naturaleza y solubilidad, principalmente carbonatos y sulfatos alcalinos y alcalinotérreos.

El problema que entraña la formación de eflorescencias, aparte del meramente estético, es la posible reducción en la impermeabilidad y durabilidad de los hormigones.

La formación de esas eflorescencias depende, fundamentalmente, del activador y de las condiciones de curado. Curados acelerados a humedades relativas superiores al $95 \%$, parecen reducir la formación de esas eflorescencias.

\section{APLICACIONES DE LOS CEMENTOS Y HORMIGONES DE ESCORIAS ACTIVADAS ALCALINAMENTE}

Dadas las características y propiedades anteriormente expuestas, se deduce que los cementos y hormigones de escorias activadas alcalinamente pueden ser utilizados prácticamente en las mismas situaciones que los hormigones de cemento portland.

Entre las aplicaciones que hasta la fecha se han dado a este tipo de materiales están: cementos para pozos petrolíferos, para la canalización de agua, para el recubrimiento de túneles para Metro y líneas ferroviarias, para bloques de prefabricados, para bases de carretera, obras portuarias, para inmovilizar residuos industriales, etc $(20,36,37)$.

Una aplicación posible y de elevado interés medioambiental, es la utilización de estos morteros y hormigones de escorias activadas alcalinamente como medio de inmovilizar residuos tóxicos y peligrosos (36, 37). En este sentido, hay trabajos en los que se recoge la inmovilización de residuos industriales, tales como compuestos de bario, clorobutadienos y fenoles (36). Malolepszy et al (37) han demostrado recientemente que los morteros de escorias activadas son capaces de retener e inmovilizar cantidades importantes de iones $\mathrm{Cd}^{2+}, \mathrm{Zn}^{2+}$ y $\mathrm{Pb}^{2+}$, con grados de inmovilización superiores, en todos los casos, al $99 \%$ en peso. Estos autores encuentran alteraciones microestructurales dependiendo de la naturaleza de los iones pesados presentes.

\section{CONSIDERACIONES FINALES}

De lo anteriormente expuesto se desprende el interés que tiene el estudio y desarrollo de estos nuevos materiales. Sin embargo, quedan todavía aspectos muy importantes por desvelar, tales como los mecanismos de hidratación y/o activación, determinar con precisión la influencia de cada uno de los factores involucrados en el proceso sobre las características de los cementos y hormigones finales, establecer los desarrollos microestructurales y las relaciones de éstos con las propiedades y características de los materiales, etc.

\section{AGRADECIMIENTOS}

Laautora agradece a laSubdirección General de Promoción de la Investigación por la subvención concedida a través del Programa Sectorial de Promoción General del Conocimiento (PB94-0048).

\section{BIBLIOGRAFÍA}

(1) PURDON, A.O. (1940): "The action of alkalis on blast-furnace slag”. J. Soc. Chem. Ind. 59, 191-202.

(2) GLUKHOVSKY, V.D.; ROSTOVSKAJA, G.S. y RUMYNA, G.V. (1980): "High strength slag-alkaline cements". TTh Intern. Congr. Chem. Cem. (Paris), 3, V-164-168.

(3) GLUKHOVSKY, V.D.; ZAITSEV, Y. y PAKHOMOW, V. (1983): "Slag-Alkaline cements and concretes-structures, properties, technological and economical askpects of the use". Silic. Ind. 10, 197-200.

(4) WANG, S. (1991): "Review of recent research on alkali-activated concrete in China”. Mag. Concr. Res. 43, № 154, 29-35.

(5) SHI, C.; WU, X y TANG, M. (1993): "Research on alkali-activated cementitious systems in China: a review”. Adv. Cem. Res. 5, $\mathrm{N}^{\circ} 17,1-7$. 
(6) GJORV, O.E. (1989): “Alkali activation of a norwegian granulated blast furnace slag”. 3rd Intern. Conf. Fly Ash, Silica Fume, Slag and Natural Pozzolans in Concrete, Trondheim, SP114-73, 1501-1517.

(7) MESTO, J. (1982): “The alkali reaction of alkali-activated finnish blast furnace slag”. Silic. Ind. 47 (4-5), 123-127.

(8) KUTTI, T. (1992): “Hydration products of alkali activated slag". IX Intern. Congr. Chem. Cem. (New Delhi), 4, 468-474.

(9) PARAMESWARAN, P.S. y CHATTERJEE, A.K. (1986): “Alkali activation of indian blast furnace slags". VIII Intern. Congr. Chem. Cem. (Rio de Janeiro), 4, 86-91.

(10) ISOZAKI, K.; IWAMOTO, S. y NAKAGAWA, K. (1986): "Some properties of alkali activated slag cement". VIII Intern. Congr. Chem. Cem. (Rio de Janeiro), 6, 395-399.

(11) CLARKE, W.J. y HELAL, M. (1994): “Alkaline activated slag and portland/slag ultrafine cements". First Intern. Conf. Alkaline cements and concretes. pp. 151-161.

(12) NARANG, K.C. y CHOPRA, S.K. (1983): "Studies on alkaline activation of BF, steel and alloy slags". Silic. Ind. 9, $175-182$.

(13) MALOLEPSZY, J. (1986): “Activation of synthetic melilite slags by alkalies”. VIII Intern. Congr. Chem. Cem. (Rio de Janeiro), 4, 104-107.

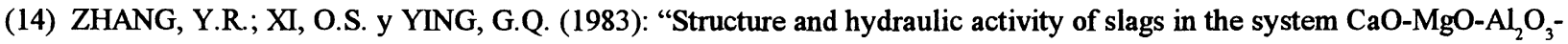
$\mathrm{SiO}_{2}$. Silic. Ind. 1, 3-6.

(15) ZHANG, Y.R.; YING, G.Q. y XI, O.S. (1988): "Study on structure and latent hydraulic activity of slag and its activation mechanism". Silic. Ind. 3/4, 55-59.

(16) SERSALE, R. (1992): Intern. Congr. Chem. Cem. (New Delhi), Vol I, Tema IIA, pp. 261-302

(17) DEMOULIAN, E.; GOURDIN, P.; HAWTHORN, F. y VERNET, C. (1980): "Influence de la composition chimique et de la texture des laitiers sur leur hydraulicité”. VII Intern. Congr. Chem. Cem. (París), vol II, pp. 89-94.

(18) SMOLCZYK, H.G. (1980): "Structure et caractérisation des laitiers". VII Intern. Congr. Chem. Cem. (París), Vol I, 1-16.

(19) SHI, C. Y LI, Y. (1989): "Investigation on some factors affecting the characteristics of alkali-phosphorous slag cement". cem. Concr. Res. Vol 19, 527-533.

(20) WANG, S.D.; SCRIVENER, K.L. y PRATT, P.L. (1994): "Factors affecting the strength of alkali-activated slag". cem. Concr, Res. Vol 24, 1033-1043.

(21) CLARKE, W.J. Y BOYD, M.D. (1994): "Ultrafine Cement strength and grouting warm springs dam". ACI Intern. Confer. Singapore, p. 141-165.

(22) HUANHAI, Z.; XUEQUAN, W.; ZHONGZI, X. y MINGSHU, T. (1993): "Kinetic study on hydration of alkali-activated slag”. Cem. Concr. Res. Vol 23, pp. 1253-1258.

(23) FERNÁNDEZ-JIMÉNEZ, A.; PUERTAS, F. y FERNÁNDEZ-CARRASCO, L: Materiales de Construcción (en prensa).

(24) WANG, S.D. y SCRIVENER, K.L. (1995): “Hydration products of alkali activated slag cement”. Cem. Concr. res. Vol 25, No 3, pp. 561-571.

(25) KRIVENKO, P.V. (1992): “Alkaline cements”. IX Intern. Congr. Chem. Cem. (New Delhi), 4, pp. 482-488. 
(26) TAYLOR, H.F.W. (1990): “Cement Chemistry”. Academic Press, London, p. 475.

(27) WANG, S.D.; PU, X.C.; SCRIVENER, K.L. y PRATT, P.L. (1995): “Alkali activated slag cement and concrete: a review of properties and problems". Adv. Cem. res. Vol 7, N²7, pp. 93-102.

(28) TAYLOR, H.F.W. (1993): "Nanostructure of C-S-H: Current status". Advn. Cem. Bas. Mat. 1, 38-46.

(29) FERNÁNDEZ-JIMÉNEZ, A. (1995): "Procesos de activación alcalino-sulfático de una escoria española de alto horno". Tesina. Universidad Autónoma de Madrid.

(30) GLASSER, F.P. (1990): “cements from micro to macrostructures”. Br. Ceram. trans. J. 89, pp. 195-202.

(31) SHI, C.; WU, XUEQUAN y TANG, M. (1991): "Hydration of alkali-slag cements at $150^{\circ} \mathrm{C}$ ". Cem. Concr. Res. Vol 21 , pp. $91-100$.

(32) BYFORS, K.; KLIGNSTEDT, G.; LEHTONEN, V.; PYY, H. y ROMBEN, L. (1989): “Durability of concrete made with alkalil activated slag”. 3rd Intern. Conf. Fly Ash, Silica Fume, Slag and Natural Pozzolans in Concrete, Trondheim, SP 114-70, 1429-1466.

(33) DEJA, J. y MALOLEPSZY, J. (1995): "Effect of carbonation on the alkali activated slag mortars and concretes". 5th Intern. Conf. Fly Ash, Silica Fume, Slag and Natural Pozzolans in Concrete, Wisconsin, Miwaukee. pp. 249-261.

(34) TAILING, B y BRANDSTETR, J, (1989): "Present state and future of alkali-activated slag concretes". 3rd Intern. Conf. Fly Ash, Silica Fume, Slag and Natural Pozzolans in Concrete, Trondheim, SP 114-74, pp. 1519-1546.

(35) DOUGLAS, E: y BILODEAU, A.; BRANDSTETR, J. Y MALHOTRA, V.M. (1991): “Alkali activated ground granulated blast furnace slag concrete: preliminary investigation". Cem. Concr. Res. Vol 21, pp. 101-108.

(36) CLARKE, W.J. y HELAL, M. (1989): “Alkali activated slag and portland/slag ultrafine cements". Material Research Society 115th Meeting. Boston, pp. 1-14.

(37) MALOLEPSZY, J. y DEJA, J. (1995): "Effect of heavy metals inmobilization on the properties of alkali activated slag mortars". 5th Intern. Conf. Fly Ash, Silica Fume, Slag, Natural Pozzolans in Concrete, Wisconssin, Milwoukee, pp. 1087-1102. 